\title{
Nature of neutrinos in the light of present and future experiments
}

\author{
M. Czakon ${ }^{1}$, J. Gluza ${ }^{1,2}$, M. Zrałek ${ }^{1}$ \\ ${ }^{1}$ Department of Field Theory and Particle Physics, Institute of Physics, \\ University of Silesia, Uniwersytecka 4, PL-40-007 Katowice, Poland \\ ${ }^{2}$ DESY Zeuthen, Platanenallee 6, 15738 Zeuthen, Germany
}

\begin{abstract}
Two general models with hierarchical and almost degenerate neutrino masses, which are able to explain the solar and atmospheric anomalies are investigated. We show how neutrinoless double beta decay experiments discern Dirac and Majorana natures of neutrinos. The strongest result is, that almost degenerate neutrinos with masses above $0.22 \mathrm{eV}$ combined with the SMA MSW must be Dirac particles. In the general case, the same bound is true for specific parity assignments. In the near future (GENIUS experiment), one of the hierarchical schemes, and the VO mechanism for almost degenerate neutrinos and specific mixing matrix elements and parity assignments will be tested.
\end{abstract}

\section{Introduction}

There are three (Weyl, Dirac and Majorana) types of spin-half fermion fields. Massless fermions are Weyl particles. Massive spin-half objects can be of either Dirac or Majorana type. All charged fermions are Dirac particles as a consequence of the electric charge conservation. Conservation of the lepton number is decidedly less fundamental than the electric charge conservation. Without lepton number conservation, neutrinos do not hold any additive internal 'charge' and can be identical to their own antiparticles. Such fermions are known as Majorana particles. The problem whether neutrinos are of Dirac or Majorana type has a long history (for a review, see e.g. [1]). The main problem is that observable effects which could differentiate between them disappear continuously with the vanishing of masses for single-handed states. This is known as the "Practical Dirac-Majorana confusion theorem" [2].

From the theoretical point of view it is quite likely that neutrinos are Majorana particles. Such objects are more fundamental and almost all extensions 
of the Standard Model predict their existence. Dirac particles can be considered as composed of two Majorana particles with opposite CP parities. But even if theory suggests that neutrinos are presumably identical to their own antiparticles, such a property should be checked experimentally.

It might be, that we reached a point where the correct answer is close.

An obvious place is the neutrinoless double $\beta$ decay, $(\beta \beta)_{o \nu}$, of nuclei. The Heidelberg-Moscow germanium experiment gives a lower limit on the half-life time [3]

$$
T_{1 / 2}^{0 \nu}>5.7 \cdot 10^{25} \mathrm{yr}(\text { at } 90 \% \text { C.L. })
$$

This result excludes an effective Majorana neutrino mass greater than $0.2 \mathrm{eV}$

$$
\left|<m_{\nu}>\right|<0.2 \mathrm{eV}
$$

The Heidelberg-Moscow collaboration proposed a new project (GENIUS) which is anticipated to be sensitive on [4]:

$$
\left|<m_{\nu}>\right| \simeq 0.01 \mathrm{eV}
$$

These bounds alone are not enough to deduce the nature of neutrinos. They give only restrictions on the combination of Majorana neutrino mixing matrix elements $U_{e i}^{2}$ and their masses $m_{i}$. However, this information can be linked to other experiments.

The connection between mass and oscillation properties of neutrinos, which follows from different experiments, has been discussed in many papers [5-10]. Howerever, most authors assume that neutrinos are Majorana particles. In this case, bounds on the $(\beta \beta)_{0 \nu}$ decay give either informations on the solar neutrino mechanism, or on the neutrino mass scale (see e.g. [6-8]). Inversely, a guess on the solar neutrino mechanism, together with a neutrino mass scale, yields $\left\langle m_{\nu}\right\rangle$ (see e.g. [9]).

Here we propose a different line of thought. The information on neutrino matrix elements and masses, given by oscillation experiments and tritium $\beta$ decay, is independent of the neutrino nature. We collect this data from the existing experiments and many phenomenological works [11], and use it to check whether bounds from negative $(\beta \beta)_{0 \nu}$ results are satisfied. If they are, neutrinos may be (but not necessarily are) Majorana particles, if not they must be Dirac particles. In this work, we discuss present (Eq. (2)), and future bounds (Eq. (3)) on the $(\beta \beta)_{0 \nu}$ decay.

In the next Section we collect all the relevant information on mixing matrix elements and masses extracted from experiments and establish two general 
mass schemes which are consistent with both solar and atmospheric data. We assume that only three massive neutrinos exist with two different and independent $\delta \mathrm{m}^{2}$. This means that we do not consider the still unsettled LSND anomaly [12] waiting for its future confirmation [13]. The main discussion is given in Section 3, where we try to infer the neutrino's character in the frame of the proposed neutrino schemes. Conclusions are to be found in Section 4.

\section{Experimental information on neutrino masses and mixing matrix elements}

Since we want to discuss both Dirac and Majorana neutrinos we must introduce appropriate mixing matrices. Without loss of generality we work in a basis where the charged lepton mass matrix is diagonal [14]. The matrix that relates the flavour Majorana states to the mass eigenstates may be parameterized by three Euler angles $\theta_{i}, \mathrm{i}=1,2,3$ and three phases $\delta, \phi_{2}$ and $\phi_{3}$ [6]. However, only one of the three phases $(\delta)$ has physical meaning to neutrino oscillations, independently of the neutrino character [14]. The two other phases $\left(\phi_{2}, \phi_{3}\right)$ enter the $(\beta \beta)_{0 \nu}$ amplitude. So, let us write the mixing matrix in a compact form (c's and s's are shortcuts for appropriate $\theta_{i}$ cosines and sines, respectively):

$$
\left(\begin{array}{c}
\nu_{e} \\
\nu_{\mu} \\
\nu_{\tau}
\end{array}\right)=\left(\begin{array}{ccc}
U_{e 1} & U_{e 2} & U_{e 3} \\
U_{\mu 1} & U_{\mu 2} & U_{\mu 3} \\
U_{\tau 1} & U_{\tau 2} & U_{\tau 3}
\end{array}\right)\left(\begin{array}{c}
\nu_{1} \\
\nu_{2} \\
\nu_{3}
\end{array}\right)
$$

where

$$
\left(\begin{array}{ccc}
U_{e 1} & U_{e 2} & U_{e 3} \\
U_{\mu 1} & U_{\mu 2} & U_{\mu 3} \\
U_{\tau 1} & U_{\tau 2} & U_{\tau 3}
\end{array}\right)=\left\{\begin{array}{cc}
U, & \text { for oscillations } \\
U V, & \text { for }(\beta \beta)_{0 \nu}
\end{array}\right.
$$

and

$$
\begin{aligned}
U & =\left(\begin{array}{ccc}
c_{1} c_{3} & c_{1} s_{3} & s_{1} e^{-i \delta} \\
-c_{2} s_{3}-s_{1} s_{2} c_{3} e^{i \delta} & c_{2} c_{3}-s_{1} s_{2} s_{3} e^{i \delta} & c_{1} s_{2} \\
-s_{2} s_{3}-s_{1} c_{2} c_{3} e^{i \delta} & -s_{2} c_{3}-s_{1} c_{2} s_{3} e^{i \delta} & c_{1} c_{2}
\end{array}\right), \\
V & =\operatorname{diag}\left(1, e^{i \phi_{2}}, e^{i\left(\phi_{3}+\delta\right)}\right) .
\end{aligned}
$$

All relevant neutrino experiments have been analyzed assuming that neutrinos oscillate. The results are the following:

- From the CHOOZ reactor [15], which measures $\bar{\nu}_{e}$ disappearance:

$$
\sin ^{2} 2 \theta_{1}<0.18 \text { for } \delta m^{2}>2 \cdot 10^{-3} \mathrm{eV}^{2}
$$


- The result of atmospheric neutrino anomaly explained by the $\nu_{\mu} \rightarrow \nu_{\tau}$ oscillation, gives [16]:

$$
\begin{aligned}
0.90 & \leq \cos ^{4} \theta_{1} \sin ^{2} 2 \theta_{2} \leq 1.0 \\
0 & \leq \sin \theta_{1} \leq 0.3
\end{aligned}
$$

with the central value of $\delta m^{2}$ :

$$
\delta m_{\text {atm }}^{2} \simeq 3.2 \cdot 10^{-3} \mathrm{eV}^{2}
$$

- Solar neutrino experiments interpreted by $\nu_{e} \rightarrow \nu_{\mu},\left(\nu_{\tau}\right)$ transitions yield [17] $\left(A_{\text {sun }}=\cos ^{4} \theta_{1} \sin ^{2} 2 \theta_{3}\right)$ :

$$
0.72 \leq A_{\text {sun }} \leq 0.95 \text { and } \delta m_{\text {sun }}^{2} \simeq 4.42 \cdot 10^{-10} \mathrm{eV}^{2}
$$

in the case of vacuum oscillation (VO);

$$
2 \times 10^{-3} \leq A_{\text {sun }} \leq 10^{-2} \text { and } \delta m_{\text {sun }}^{2} \simeq 5 \cdot 10^{-6} \mathrm{eV}^{2}
$$

in the case of small mixing MSW transition (SMA MSW) and

$$
0.65 \leq A_{\text {sun }} \leq 1.0 \text { and } \delta m_{\text {sun }}^{2} \simeq 2 \cdot 10^{-5} \mathrm{eV}^{2}
$$

in the case of large mixing MSW transition (LMA MSW).

Direct kinematical measurement of the $\bar{\nu}_{e}$ mass from the tritium $\beta$ decay yield:

$$
m\left(\nu_{e}\right)=\left[\left|U_{e 1}\right|^{2} m_{1}+\left|U_{e 2}\right|^{2} m_{2}+\left|U_{e 3}\right|^{2} m_{3}\right]<m_{\beta}
$$

where values of $m_{\beta}$ differ slightly between the two collaborations, namely:

$$
m_{\beta}=3.4 \mathrm{eV}[18], \quad m_{\beta}=2.7 \mathrm{eV}[19] .
$$

Combining experimental constraints from atmospheric and solar neutrino oscillations with the tritium $\beta$ decay limit (Eq.(11)) it is possible to infer interesting limits on the highest mass eingenvalue and on mass differences.

For three neutrino eigenmasses

$$
m_{1}<m_{2}<m_{3}
$$

it was found [20]:

$$
\begin{gathered}
\sqrt{\delta m_{a t m}^{2}} \leq m_{3} \leq \sqrt{m_{\beta}^{2}+\delta m_{a t m}^{2}} \\
\left|m_{i}-m_{j}\right|<\sqrt{\delta m_{a t m}^{2}}, \quad i, j=1,2,3
\end{gathered}
$$




\section{Scheme I}

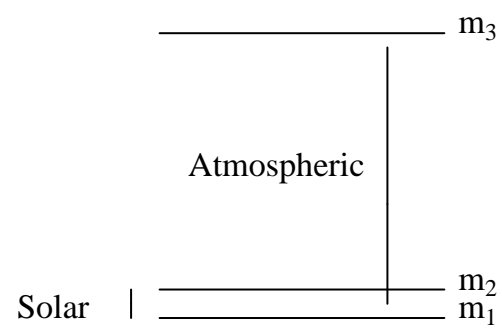

Scheme II

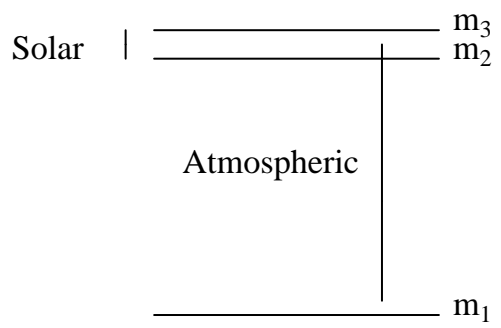

Fig. 1. Two possible neutrino mass spectra which can describe the oscillation data. Scheme I describes the atmospheric anomaly with $\delta m_{31}^{2} \simeq \delta m_{32}^{2}=\delta m_{\text {atm }}^{2}$ and solar neutrino oscillations with $\delta m_{21}^{2}=\delta m_{\text {sun }}^{2}$. Scheme II describes the atmospheric anomaly with $\delta m_{31}^{2} \simeq \delta m_{21}^{2}=\delta m_{a t m}^{2}$ and solar neutrino oscillations with $\delta m_{32}^{2}=\delta m_{\text {sun }}^{2}$.

For the present experimental values $\left(\delta m_{a t m}^{2} \simeq(1.5 \div 6.0) \cdot 10^{-3} \mathrm{eV}^{2}(90 \%\right.$ C.L.) [16] we have:

$$
\begin{aligned}
0.04 \mathrm{eV} & <m_{3}<2.7 \mathrm{eV}, \\
\left|m_{i}-m_{j}\right| & <0.08 \mathrm{eV}, \quad i, j=1,2,3 .
\end{aligned}
$$

These constraints are satisfied by two possible mass spectra given in Fig.1. In addition the total scale for neutrino masses is not fixed.

The lowest mass $\mathrm{m}_{1}$ can be close to zero or much higher, up to $2.7 \mathrm{eV}$.

(1) In the first case the mass spectrum can be hierarchical with $m_{3}>>m_{2}>$ $m_{1}$ (Fig.1; scheme I) or $m_{3}>m_{2}>>m_{1}$ (Fig1; scheme II).

(2) In the second situation, masses are almost degenerate $m_{1} \simeq m_{2} \simeq m_{3}$, but schemes I and II, which describe solar and atmospheric anomalies, are the same. Since cosmological estimations give [21]

$$
\sum_{\nu} m_{\nu} \leq 5 \mathrm{eV}
$$

all three neutrinos have masses close to (or below) $1.7 \mathrm{eV}$.

\section{Constraints on the nature of neutrinos}

Oscillations of Dirac and Majorana neutrinos share the same description. Bounds on mixing angles Eqs.(5-10) do not feel their character, contrary to the effective mass (Eq. $(2,3))$. For a Dirac neutrino $<m_{\nu}>=0$. For a Majorana 
neutrino [22]:

$$
\left|<m_{\nu}>\right|=\left.|| U_{e 1}\right|^{2} m_{1}+\left|U_{e 2}\right|^{2} m_{2} e^{2 i \phi_{2}}+\left|U_{e 3}\right|^{2} m_{3} e^{2 i \phi_{3}} \mid .
$$

The $\mathrm{U}$ matrix is unitary, so only two elements of $\left|U_{e i}\right|$ are independent.

Let us bound $\left|U_{e 2}\right|^{2}$ and $\left|U_{e 3}\right|^{2}$. From Eqs.(5) and (6) we can find $\left(x=\cos ^{2} \theta_{1}\right)$

$$
0 \leq\left|U_{e 3}\right|^{2} \equiv(1-x) \leq 0.05 \text {. }
$$

The constraints on $\left|U_{e 2}\right|^{2}$ are obtained from Eqs.(8),(9) and (10). As the $A_{\text {sun }}$ amplitude depends on $\sin ^{2} 2 \theta_{3}, \sin ^{2} \theta_{3}$ and $\cos ^{2} \theta_{3}$ are not fixed independently. Two values for $\theta_{3}$ in the range $0<\theta_{3}<\frac{\pi}{4}$ and $\frac{\pi}{4}<\theta_{3}<\frac{\pi}{2}$ can be inferred from bounds on $\sin ^{2} 2 \theta_{3}$, giving two possible values of $\left|U_{e 2}\right|^{2}$. We will call them small and large $\sin ^{2} \theta_{3}$.

The vacuum oscillation Eq.(8) yields:

(1) For small $\sin ^{2} \theta_{3}$ :

$$
\frac{x}{2}\left(1-\sqrt{1-\frac{0.72}{x^{2}}}\right) \leq\left|U_{e 2}\right|_{S}^{2(V O)} \leq\left\{\begin{array}{cl}
\frac{x}{2} & 0.9 \leq x^{2} \leq 0.95 \\
\frac{x}{2}\left(1-\sqrt{1-\frac{0.95}{x^{2}}}\right) & 0.95 \leq x^{2} \leq 1.0
\end{array}\right.
$$

(2) For large $\sin ^{2} \theta_{3}$ :

$$
\frac{x}{2}\left(1+\sqrt{1-\frac{0.72}{x^{2}}}\right) \geq\left|U_{e 2}\right|_{L}^{2(V O)} \geq\left\{\begin{array}{cl}
\frac{x}{2} & 0.9 \leq x^{2} \leq 0.95 \\
\frac{x}{2}\left(1+\sqrt{1-\frac{0.95}{x^{2}}}\right) & 0.95 \leq x^{2} \leq 1.0
\end{array}\right.
$$

Therefore, $0.95 \leq x \leq 1.0$ leads to:

$$
\begin{aligned}
& 0.24 \leq\left|U_{e 2}\right|_{S}^{2(V O)} \leq 0.48 \\
& 0.48 \leq\left|U_{e 2}\right|_{L}^{2(V O)} \leq 0.76
\end{aligned}
$$

In a similar way, bounds from the SMA and LMA solutions to the solar anomaly are:

(1)

$$
\begin{gathered}
0.0005 \leq\left|U_{e 2}\right|_{S}^{2(S M A)} \leq 0.0026 \\
0.947 \leq\left|U_{e 2}\right|_{L}^{2(S M A)} \leq 0.999
\end{gathered}
$$


(2)

for SMA MSW, and:

$$
\begin{gathered}
0.204 \leq\left|U_{e 2}\right|_{S}^{2(L M A)} \leq 0.48, \\
0.48 \leq\left|U_{e 2}\right|_{L}^{2(L M A)} \leq 0.8
\end{gathered}
$$

for LMA MSW.

Equipped with bounds on $\left|U_{e 2}\right|^{2}$ and $\left|U_{e 3}\right|^{2}$, we are able to explore the meaning of Eqs. $(2,3)$.

\subsection{Hierarchical neutrino mass scenario}

First, let us consider the mass scheme I from Fig.1. Since $m_{1} \simeq 0$ :

$$
\begin{aligned}
& m_{2}=\sqrt{\delta m_{\text {sun }}^{2}}= \begin{cases}2.1 \cdot 10^{-5} \mathrm{eV} & (V O) \\
2.2 \cdot 10^{-3} \mathrm{eV} & (S M A) \\
4.5 \cdot 10^{-3} \mathrm{eV} & (L M A),\end{cases} \\
& m_{3}=\sqrt{\delta m_{\text {atm }}^{2}}=0.057 \mathrm{eV}
\end{aligned}
$$

Using the value of $m_{3}$, the experimental data on $(\beta \beta)_{0 \nu}$ translate into the following:

$$
\frac{\left|<m_{\nu}>\right|}{m_{3}}=\left.|| U_{e 2}\right|^{2} \frac{m_{2}}{m_{3}}+\left|U_{e 3}\right|^{2} e^{2 i\left(\phi_{2}-\phi_{3}\right)} \mid \leq\left\{\begin{array}{cc}
3.5 & \text { (present bound) } \\
0.18 & \text { (GENIUS) }
\end{array}\right.
$$

On the other hand, even for the large $\sin ^{2} \theta_{3}$ solution, where $U_{e 2} \simeq 1$, we get:

$$
\left(\frac{\left|<m_{\nu}>\right|}{m_{3}}\right)_{\max }=\frac{m_{2}}{m_{3}}+\left|U_{e 3}\right|^{2} \leq 0.13 .
$$

This means that even the future sensitivity of GENIUS will not be enough to discern neutrino nature.

Let us explore now the hierarchical mass scheme II from Fig.1:

$$
\begin{aligned}
& m_{2}=\sqrt{\delta m_{a t m}^{2}}=0.057 \mathrm{eV} \\
& m_{3}=\sqrt{\delta m_{a t m}^{2}+\delta m_{\text {sun }}^{2}} \simeq 0.057 \mathrm{eV} .
\end{aligned}
$$


This again can be transformed into:

$$
\frac{\left|<m_{\nu}>\right|}{m_{3}}=\left.|| U_{e 2}\right|^{2}+\left|U_{e 3}\right|^{2} e^{2 i\left(\phi_{2}-\phi_{3}\right)} \mid \leq\left\{\begin{array}{cc}
3.5 & \text { (present bound) } \\
0.18 & \text { (GENIUS) }
\end{array}\right.
$$

From unitarity of the U matrix follows that the present bound is always satisfied. The result of GENIUS is much more interesting. We can find the minimal values of the effective mass

$$
\left(\frac{\left|<m_{\nu}>\right|}{m_{3}}\right)_{\min }=\left.|| U_{e 2}\right|^{2}-\left|U_{e 3}\right|^{2} \mid
$$

namely:

$$
\left(\frac{\left|<m_{\nu}>\right|}{m_{3}}\right)_{\text {min }}=\left\{\begin{array}{cc}
0.21 & \text { VO, small } \sin ^{2} \theta_{3}, \\
0.42 & \text { VO, large } \sin ^{2} \theta_{3}, \\
<0.05 & \text { SMA, small } \sin ^{2} \theta_{3} \\
0.9 & \text { SMA, large } \sin ^{2} \theta_{3} \\
0.18 & \text { LMA, small } \sin ^{2} \theta_{3} \\
0.43 & \text { LMA, large } \sin ^{2} \theta_{3}
\end{array}\right.
$$

For VO and LMA MSW solutions of the solar neutrino problem, a negative result of the GENIUS experiment would mean that neutrinos must be Dirac particles, since our bounds exceed those infered by this collaboration. For the SMA MSW solution, conclusions depend on the values of $\sin ^{2} \theta_{3}$. Next experiments (SNO, BOREXINO) which will be able to discern types of oscillations can give informations on $\sin ^{2} \theta_{3}$.

\subsection{Degenerate neutrino mass scenario}

We assume that neutrino mass is much larger than $\sqrt{\delta m_{a t m}^{2}}$. Let it be that $\sum_{\nu} m_{\nu} \simeq 5 \mathrm{eV}$, so $m_{\nu} \simeq 1.7 \mathrm{eV}$. Now, the $(\beta \beta)_{0 \nu}$ bound has the form:

$$
\frac{\left|<m_{\nu}>\right|}{m_{\nu}}=\left.|| U_{e 1}\right|^{2}+\left|U_{e 2}\right|^{2} e^{2 i \phi_{2}}+\left|U_{e 3}\right|^{2} e^{2 i \phi_{3}} \mid \leq\left\{\begin{array}{cc}
0.12 & \text { (present bound) } \\
0.006 & \text { (GENIUS) }
\end{array}\right.
$$

The discussion is more difficult, since we do not have any information about the $\mathrm{CP}$ violating phases $\phi_{1}, \phi_{2}$. So, let us assume that $\mathrm{CP}$ is conserved and consider all possible $C P= \pm i\left(e^{i \phi_{i}}= \pm 1\right)$ phases for neutrinos. As only relative $\mathrm{CP}$ phases have physical consequences, we take $\eta_{C P}\left(\nu_{1}\right)=+i$. There 
are four different arrangements: (A) $\eta_{C P}\left(\nu_{1}\right)=\eta_{C P}\left(\nu_{2}\right)=\eta_{C P}\left(\nu_{3}\right)=+i$, (B) $\eta_{C P}\left(\nu_{1}\right)=-\eta_{C P}\left(\nu_{2}\right)=-\eta_{C P}\left(\nu_{3}\right),(\mathrm{C}) \eta_{C P}\left(\nu_{1}\right)=\eta_{C P}\left(\nu_{2}\right)=-\eta_{C P}\left(\nu_{3}\right)$ and (D) $\eta_{C P}\left(\nu_{1}\right)=-\eta_{C P}\left(\nu_{2}\right)=\eta_{C P}\left(\nu_{3}\right)$. In the case, CP is broken, the condition (34) looks more complicated, but qualitatively our discussion will not change. The four CP conserving cases give extreme values for $\left|<m_{\nu}\right\rangle \mid$, namely:

$$
\begin{array}{ll}
(A): & \frac{<m_{\nu}>}{m_{\nu}}=1, \\
(B): & \frac{<m_{\nu}>}{m_{\nu}}=\left.|1-2| U_{e 2}\right|^{2}-2\left|U_{e 3}\right|^{2} \mid, \\
(C): & \frac{<m_{\nu}>}{m_{\nu}}=\left.|1-2| U_{e 3}\right|^{2} \mid, \\
(D): & \frac{<m_{\nu}>}{m_{\nu}}=\left.|1-2| U_{e 2}\right|^{2} \mid
\end{array}
$$

Taking all possible values of the mixing matrix elements (Eqs. (17)-(25)) several interesting conclusions come to mind.

1) If neutrinos are Majorana particles and $\mathrm{CP}$ is conserved, already the present bound (Eq.(34)) is not satisfied in the cases (A) and (C) for $m_{\nu}=1.7 \mathrm{eV}$, independently of the oscillation mechanism for the solar neutrino deficit. The lower bound on the masses for which this statement is true is approximately $0.22 \mathrm{eV}$. This means that almost degenerate neutrinos cannot have the same $\mathrm{CP}$ parities [7], and the CP parity of the heaviest neutrino cannot be opposite to the $\mathrm{CP}$ eigenvalues of the lighter ones.

Let us discuss cases (B) and (D).

2) For the vacuum oscillation mechanism, the only interesting possibility is the (D) case, where $\left(\frac{\left|\left\langle m_{\nu}\right\rangle\right|}{m_{\nu}}\right)_{\text {min }} \simeq 0.040$ for small $\sin ^{2} \theta_{3}$. Other oscillation scenarios, namely with large $\sin ^{2} \theta_{3}$ and the case (B) allow $\left(\frac{\left|\left\langle m_{\nu}\right\rangle\right|}{m_{\nu}}\right)_{\min } \simeq 0$.

3) For SMA MSW, both cases (B) and (D) give a minimal value of $\frac{\left\langle m_{\nu}\right\rangle}{m_{\nu}}$ greater than 0.9. The present bound (Eq.(34)) is not satisfied, neutrinos would have to be Dirac particles. Or, by opposite, if neutrinos are almost degenerate Majorana particles with $m_{\nu}>0.22 \mathrm{eV}$, the SMA MSW mechanism is not a proper explanation of the solar neutrino deficit [8].

4) The LMA MSW case is similar to the VO case, because the ranges of the U matrix elements are comparable (Eqs.(20)-(21) and Eqs.(24-25)). 


\section{Conclusions}

We have combined data on solar and atmospheric neutrino oscillations with the present and future bounds on $(\beta \beta)_{0 \nu}$.

Present $(\beta \beta)_{0 \nu}$ experiments imply:

- None of the hierarchical schemes I and II is able to help us to determine the nature of neutrinos.

- A degenerate scheme with Majorana neutrinos of (A) or (C) parities is excluded if $m_{\nu}>0.22 \mathrm{eV}$.

- Degenerate Majorana neutrinos with $m_{\nu}>0.22 \mathrm{eV}$ and both (B),(D) parity assignments combined with the SMA MSW are excluded.

The future $(\beta \beta)_{0 \nu}$ experiment GENIUS gives a chance to see whether:

- Majorana neutrinos of the hierarchical scheme II fit the data, which is not the case of scheme I;

- the VO mechanism with degenerate neutrinos, small $\sin ^{2} \theta_{3}$ and (D) parity assignments is correct.

\section{Acknowledgements}

This work was supported by Polish Committee for Scientific Research under Grants Nos. 2P03B08414 and 2P03B04215. J.G. would like to thank the Alexander von Humboldt-Stiftung for a fellowship.

\section{References}

[1] M. Zrałek, Acta Phys. Pol. 28 (1997) 2225.

[2] L.F. Li, F. Wilczek, Phys. Rev. D25 (1982) 143; B. Kayser, R.E. Schrock, Phys. Lett. B112 (1982) 137;y B. Kayser, Phys. Rev. D26 (1982) 1662.

[3] L. Baudis et. al., hep-ex/9902014.

[4] H.V. Klapdor-Kleingrothaus, J. Hellmig, M. Hirsch, J. Phys. G24 (1998) 484; J. Hellmig, H.V. Klapdor-Kleingrothaus, Z. Phys. A359(1997)351; H.V. KlapdorKleingrothaus, M. Hirsch, Z. Phys. A359(1997)361; C.E. Aalseth et. al., Nucl. Phys. (Proc. Suppl.) 70 (1999) 236; X. Sarazin (NEMO Coll.) ibid. 70 (1999) 239; V.D. Ashitkov et. al., ibid. 70 (1999) 233; E. Fiorini, Phys. Rep. 307 (1998) 309; H.V. Klapdor-Kleingrothaus, hep-ex/9907040. 
[5] S.T. Petcov, A. Yu. Smirnov, Phys. Lett. B322 (1994) 109; S.M. Bilenky, A. Bottino, C. Giunti, C.W. Kim, Phys. Lett. B356 (1995) 273; S.M. Bilenky, C. Giunti, C.W. Kim, M. Monteno, Phys. Rev. D57 (1998) 6981; S.M. Bilenky, C. Giunti, W. Grimus, hep-ph/9809368; F. Vissani, hep-ph/9708483; hepph/9904349; hep-ph/9906525;

[6] V. Barger and K. Whisnant, hep-ph/9904281.

[7] R. Adhikair, G. Rajasekaran, hep-ph/9812361.

[8] H. Georgi, S.L. Glashow, hep-ph/9808292 H. Minakata, O. Yasuda, Phys. Rev. D56 (1997) 1692.

[9] S.M. Bilenky, C. Giunti, hep-ph/9904328; C. Giunti, hep-ph/9906275. S.M. Bilenky, C. Giunti, W. Grimus, B. Kayser, S.T. Petcov, hep-ph/9907234.

[10] G.C. Branco, M.N. Rebelo, J.I. Silva-Marcos, Phys. Rev. Lett. 82 (1999) 682.

[11] see review papers:

S.M. Bilenky, C. Giunti, W. Grimus, hep-ph/9812360; Europhysics Neutrino Oscillation Workshop 98, hep-ph/9906251; P. Fisher, B. Kayser, K.S. McFarland, hep-ph/9906244; J. Ellis, hep-ph/9907458.

[12] C. Athanassopoulos et al., Phys. Rev. Lett. 77(1996)3082; Phys. Rev. C54(1996)268; Phys. Rev. C58(1998)2489; Phys. Rev. Lett. 81(1998)1774; S.J. Yellin, hep-ex/9902012.

[13] B. Zeitnitz et al., Prog. Part. Nucl. Phys., 40 (1998) 169; B. Armbruster et. al., Phys. Rev. C57 (1998) 3414; E. Eitel and B. Zeitnitz, hep-ex/9809007; for MiniBooNE see http://www.neutrino.lanl.gov/BooNE/.

[14] F. del Aquila, M. Zrałek, Nucl. Phys. B447(1995)211; Acta Phys. Pol. B27 (1996) 971.

[15] CHOOZ Collaboration, M. Apollonio et. al., Phys. Lett. B420(1998)397.

[16] K. Scholberg, hep-ex/9905016.

[17] M.B. Smy, hep-ex/9903034; J.N. Bahcall, P.I. Krastev, A. Yu. Smirnow, Phys. Rev. D58(1998)096016; hep-ph/9905220.

[18] C. Weinheimer (Mainz), Talk at Ringberg Euroconference "New Trends in Neutrino Physics", May 1998; H. Berth et al., Prog. Part. Nucl. Phys. 40 (1998) 353; C. Weinheimer et. al., Phys. Lett. B300 (1993) 210.

[19] V.M. Lobashev (Troitsk), Talk at Ringberg Euroconference "New Trends in Neutrino Physics", May 1998; V.M. Lobashev, Prog. Part. Nucl. Phys. 40 (1998) 337; A.I. Belesev et.al., Phys. Lett. B350 (1995) 263.

[20] V. Barger, T.J. Weiler, K. Whisnant, Phys. Lett. B442(1998)255.

[21] see e.g. G.G. Raffelt, hep-ph/990271; G. Gelmini, hep-ph/9904369.

[22] see e.g. M. Doi, T. Kotani, E. Takasugi, Prog. Theor. Phys. (supplement) 83 (1985) 1. 